\title{
Emergence Is Why It Is Not Possible to Explain Life Solely with Physics and Chemistry
}

\author{
Vincent Vesterby
}

There is a longstanding belief that it is possible to understand life and mind solely with the knowledge of physics and chemistry. While a human body, and the life and mind that occurs therein, are composed of molecules and molecular interactions, it is physically impossible for life and mind to be the products of molecular interactions alone. There is something more - emergent pattern of material organization and hierarchic organization above the level of molecular structure and interaction.

That which is more, and which intrinsically defines the nature of life and mind, is a consequence of two general foundational factors of the intrinsic existential and organizational nature of reality. These factors are the occurrence of emergence and that the whole is invariably greater than the sum of the parts. Emergence is important here because it is the general process by which developed stages of organization and higher levels of hierarchy come into being. It is the process whereby the whole comes into existence. That the whole is greater than the sum of the parts is important because that is why there is something more. What it is that is more is a new, more developed, pattern of material organization - and the roles it plays in the further development of a system and in hierarchic structure.

New pattern of material organization is the consequence of emergence. When parts come together, they form patterns, patterns of parts and the relations between them. The pattern of the parts and their interrelations constitutes the pattern of the group, the pattern of the whole. The whole is a pattern, a configuration. As such it plays roles in relation to other wholes, other configurations.

The roles a whole plays in the formation of a more complex stage of combination or a new hierarchic level are determined by what it is, by its configuration, by how its parts are arranged, and by the manner in which it comes together with some other unit of matter of appropriate configuration. The configuration of the consequent, newly existing, emergent whole is determined by the configurations of its components and their manner of coming together because those are the factors that are-there to play the determining roles.

There are three levels of hierarchic structural organization here, and two stages of emergent pattern of material organization.

First, at the bottom level of the hierarchic structure, there are the initial components, which come together with the consequent emergence (first case) of the components of the second level of the hierarchic structure. When these components of the second level come together there is the consequent emergence (second case) of the components of the third level of the hierarchic structure. 
There are two kinds of existential-dependency playing roles in this situation-one occurs in relation to time and the other in relation to space.

Earlier stages of the developmental process that creates a system are required for the current existence of the system. Those earlier stages might or might not still be present in the system, but the existence of the current stage of the system is existentially-dependent on the prior occurrence of the earlier stages. If those stages did not exist and play their roles, the system would not now exist. In the previous paragraph, the occurrence of the second stage of emergence is existentially-dependent on the occurrence of the first stage - a temporal existential-dependency relation.

Lower levels of a hierarchical structure are required for the existence of the higher levels. In the hierarchical structure of an object or system, lower levels are still present and playing their roles, and the existence of the higher levels is existentially-dependent on the presence and roles of those lower levels. If those lower levels did not exist, the hierarchic structure would not exist - a structural existential-dependency relation organized in space.

That which follows is existentially-dependent on that which has gone before, developmentally and structurally. The existence and nature of that which goes before determines the existence and nature of that which follows. What goes before determines what follows for the specific reason that what goes before is what is-there to play that role.

Reality is organized hierarchically, and the configuration of each level, its pattern of organization, enables the existence of the higher levels. The configuration of elementary particles enables the existence of atoms, the configuration of atoms enables the existence of molecules, the configuration of molecules enables the existence of tissues, the configuration of tissues enables the existence of organs, the configuration of organs enables the existence of organ systems, and the configuration of organ systems enables the existence of organisms.

This is, of course, a limited description of the situation, with the reality being much more complex. Real hierarchic situations are usually compound and complexly interrelated. As a simple example, the situation of two molecules joined together is a combination of two separate hierarchies, and they interrelate at both the highest level, by way of the configuration of each molecule, and at lower levels of their hierarchic structures, by way of the bonds that actually join them together. The human body is extravagantly compound and complex in this manner, structurally and functionally.

Each level is something more. An atom is more than an elementary particle. A molecule is more than an atom. A tissue is more than a molecule, and so on. The whole is more than the sum of the parts because it is composed of both the parts and their interrelations. The whole is the pattern of organization of the parts, their combined configuration. And that configuration plays roles that are beyond those of its components. 
Everything about any system that is composed of molecules is a product of molecular interactions - plus the emergent configurations, and the emergent roles of the configurations, of all the emergent stages and emergent levels between the molecules and the configuration of the whole system. You cannot achieve a full understanding of the relation between two stages or levels that are twice removed from one another without understanding the role of the intervening stage or level. Just as an organism, and life, and mind, emerge developmentally and structurally stage by stage and level by level, a full, deep, understanding of life and mind must incorporate that emergent development, stage by stage, and that emergent structure, level by level.

This paper discusses aspects of the role of emergence in the universe, and the importance of understanding those roles for the purpose of understanding life and mind. There are two other papers that provide knowledge about the intrinsic nature of emergence and about how understanding emergence as an isomorphy makes it possible to achieve transdisciplinary understanding of the universe.

The Intrinsic Nature of Emergence-With Illustrations.

Vesterby, Vincent. 2019. "The Intrinsic Nature of Emergence-with Illustrations." OSF Preprints. July 9. osf.io/n2skj.

\section{Emergence Is an Isomorphy}

Vesterby, Vincent. 2019. "Emergence Is an Isomorphy.” OSF Preprints. July 4. doi:10.31219/osf.io/ys3wd.

Date of original version of "Emergence Is Why It Is Not Possible to Explain Life Solely with Physics and Chemistry"-June 2017. 